\title{
Type III Hypersensitivity
}

National Cancer Institute

\section{Source}

National Cancer Institute. Type III Hypersensitivity. NCI Thesaurus. Code C114346.

A hypersensitivity reaction resulting from the deposition of antigen-antibody immune complexes in tissues, which trigger activation of the complement system. 